\title{
A Novel Defined Ferroptosis-Related Gene Signature for Predicting The Prognosis of Kidney Renal Clear Cell Carcinoma
}

\author{
Ke-Hao Pan \\ Zhongda Hospital Southeast University \\ Yi-Fan Liu \\ Southeast University \\ Bin Xu \\ Zhongda Hospital Southeast University \\ Ming Chen ( $\sim$ pankehao0319@126.com ) \\ Zhongda Hospital Southeast University
}

\section{Research Article}

Keywords:

Posted Date: January 19th, 2022

DOI: https://doi.org/10.21203/rs.3.rs-882590/v2

License: (9) (1) This work is licensed under a Creative Commons Attribution 4.0 International License. Read Full License 


\section{Abstract}

Objective: We developed a predictive model associated with ferroptosis to provide a more comprehensive view of kidney renal clear cell carcinoma (KIRC) immunotherapy.

Methods: Gene expression data and corresponding clinical outcomes were obtained from the GEO and The Cancer Genome Atlas (TCGA) databases, and a ferroptosis-related gene set was obtained from the FerrDb database.

Results: We identified 17 ferroptosis-related genes that were differentially expressed, including enrichment in genes involved in the immune system process. We established a ferroptosis-related gene-based prognostic model based on the results of univariate Cox regression and multivariate Cox regression analyses, with an area under the curve (AUC) of 0.644 (3 years). We found that the higher exprssion of MT1G, LAMP2 and MIOX showed a higher proportion of CD8+ T cells, CD4+ memory activated T cells, etc. Finally, a predictive ferroptosis-related prognostic nomogram, which included the predictive values of age, gender, grade, TNM stage, and risk score, was established to predict overall survival.

Conclusions: In sum, we developed a ferroptosis-related gene-based prognostic model that provides novel insights into the prediction of KIRC prognosis and identifies the relevance of the immune microenvironment for patient outcomes.

\section{Introduction}

Renal cell carcinoma(RCC) is one of the most common malignant tumors of the urinary system, after bladder cancer, accounting for $46.3 \%$ of all new cancer cases of the urinary system. According to the cancer statistics, there will be about 73,750 new cases and 14,830 new deaths in the United States in $2020^{[1]}$. Kidney renal clear cell carcinoma(KIRC) is the most common subtype of renal cell carcinoma, representing $70-85 \%{ }^{[2]}$. At present, lots of risk factors associated with the occurrence and progression of KIRC have been reported, such as smoking ${ }^{[3]}$, obesity ${ }^{[4]}$, alcoho ${ }^{[5]}$, hypertension ${ }^{[6]}$, occupational exposure and drugs ${ }^{[7-8]}$. Although great breakthroughs have been made in the diagnosis, treatment and prognosis of KIRC, the prognosis of patients with KIRC is still unsatisfactory ${ }^{[9]}$, with about $30 \%$ of patients have tumor recurrence after being considered disease-free ${ }^{[10-11]}$. In 2020, about 179,368 patients died of RCC ${ }^{[12]}$. Currently, the pathogenesis of KIRC is unknown, and no sensitive tumor biomarkers have been identified. Therefore, there is an urgent need to identify effective therapeutic targets or to find promising prognostic biomarkers in KIRC.

Cell death plays an important role in maintaining normal development homeostasis and inhibiting excessive proliferation of tumor cells ${ }^{[13]}$. It can be divided into two categories: accidental cell death (ACD) and regulated cell death (RCD). RCD mainly includes apoptosis, programmed necrosis, pyrosis and ferroptosisis. The main characteristics of ferroptosisis are the accumulation of ferrous ions and the aggregation of lipid peroxides on the membrane ${ }^{14]}$. Ferroptosisis not only participates in some pathological processes as a stress response ${ }^{[15-16]}$, but also contributes to the anticancer effect of some tumor suppressors (such as BaP1 and p53) ${ }^{[17-18]}$. It is highly sensitive to ferroptosisis in tumor cells with inherent or acquired drug resistance ${ }^{[19-20]}$, and is closely related to the effect of tumor immunotherapy ${ }^{[21]}$. Whether ferroptosisis can participate in the body's 
development and normal physiological process as programmed death such as apoptosis and autophagy is still

under active research, but there are many reports about the potential role of ferroptosisis in tumor ${ }^{[22-23]}$. Most tumors are in a state of high oxidative stress, so that tumor cells also need to increase the scavenging capacity of ROS to prevent oxidative damage. Therefore, most tumor cells (such as hepatocellular carcinoma, osteosarcoma, prostate cancer, ovarian cancer, etc.) are more vulnerable to iron death ${ }^{[24]}$. Moreover, ferroptotic cancer cells might release signals like oxidized lipid mediators to affect anti-tumor immunity, or a small proportion of cells undergoing ferroptosis might suppress the immune system and allow tumor growth ${ }^{[25]}$.

Given the existing findings, we know that ferroptosisis plays an important role in the development of tumours and antitumour processes; however, its specific functions in KIRC have been less studied. Thus, we performed a systematic study to determine the expression levels of pyroptosis-related genes between normal renal and KIRC tissues, explore the prognostic value of these genes, and study the correlations between pyroptosis and the tumour immune microenvironment.

\section{Results}

\section{Differentially expressed ferroptosis signatures in KIRC}

The information of GEO datasets used is listed in Table 1. Following the differential gene analysis, 1072 dysregulated genes were obtained from GEO: GSE168845, with 422 genes showing upregulation and 650 showing downregulation, and 572 dysregulated genes from GEO: GSE105261, with 194 genes showing upregulation and 380 showing downregulation. Finally, we obtained 886 dysregulated genes from GEO: GSE11151, of which 343 genes were upregulated and 543 genes were downregulated (Figure 1A). Because the ferroptosis-related genes obtained from the FerrDb database were experimentally validated, the differentially expressed genes (DEGs) obtained from the GEO datasets were intersected with the ferroptosis-gene set to obtain "differentially expressed ferroptosis genes". The Venn diagram revealed that 17 ferroptosis-related genes intersected between four datasets (Figure 1A).

To explore the underlying mechanisms of the ferroptosis signatures in $\mathrm{KIRC}$, we performed a functional analysis. As shown in Figures 1B and Table 2, the Gene Ontology (GO) analysis results suggest that these dysregulated ferroptosis genes are mainly enriched in iron ion binding,microvillus membrane and regulation of transcription from RNA polymerase II promoter in response to stress. Furthermore, the protein-protein interaction (PPI) network and MCODE plugin identified the significant modules in these ferroptosis genes(Figure 1D).

\section{The establishment and verification of a ferroptosis-related prognostic model}

Normalized mRNA expression data with corresponding patients' information were obtained from TCGA-KIRC. Univariate Cox regression analysis was first applied to detect the genes that were significantly associated with prognosis. As shown in Figures 2A-2G, seven ferroptosis-related genes were identified as prognosis genes. Meanwhile, expression analysis of the seven ferroptosis genes with prognostic value in KIRC samples compared with normal tissues was made (Figure $3 A-G$ ). A forest plot showing the results of the univariate Cox regression analysis is shown in Figure $2 \mathrm{H}$. MT1G, PROM2, LAMP2 and MIOX were subsequently identified as independent prognostic signatures in a multivariate Cox regression (Table 3). Thus, a prognostic model was 
established based on the multivariate Cox regression. As shown in Figure 3, a risk score for each patient was calculated as follows: lambda. $\min =0.0206$, Riskscore $=(0.0563) \star M T 1 G+(-0.2932) \star L A M P 2+(-0.1039) \star M I O X$. Then, a high-risk group and a low-risk group were stratified based on the median of the risk score. Moreover, $t-$ distributed stochastic neighbor embedding(t-SNE) and principal-component analysis(PCA) showed that the patients in different groups were distributed in two directions. The median survival time of patients with a high

risk score was significantly shorter than that of the patients with a low risk score (Figure 4B). Subsequently, we created a receiver operating characteristic curve (ROC) to evaluate the prognosis prediction efficiency of the model, and we found the area under the curve (AUC) was 0.676 (1-year OS), 0.644 (3-year 0S), and 0.674 (5year OS), indicating the predictive model was well established (Figure 3C). Finally, MT1G, LAMP2 and MIOX was considered as prognostic value genes.

\section{Construction of the nomogram}

A predictive ferroptosis-related prognostic nomogram was established using the results of the multivariate analysis. To identify the predictive value of age, gender, grade, TNM stage, and risk score, we used nomograms to predict the 1-, 3-, and 5-year OS (Figure 5D). As shown, this nomogram was able to assess several variables to predict a patient outcome, which is based on patient characteristics, including age, gender, TNM stage, and risk score. Additionally, the predictive accuracy for OS is shown by the calibration curves. A calibration curve for the predictive probability showed an accordant agreement for the 3-year OS (Figure 5E).

To explore the possible relationships between MT1G, LAMP2 and MIOX and multiple clinicopathologic factors, correlation analysis was conducted via independent $t$ tests. These fndings demonstrated that MT1G, LAMP2 and MIOX was statistically correlated with multiple clinicopathological factors(Figure 5A-C).

\section{Correlation between risk score of ferroptosis-related-DEGs risks prognostic model and immune score}

To further investigate the relationship between the ferroptosis-related-DEGs risks prognostic model and expression levels at the immune cell level, Spearman correlation analysis was made between risk score and immune score. The results(Figure 6) showed that the risk score was associated with expression of macrophage $M 1(p=002)$, monocyte $(p=0.001)$, and neutrophil $\left(p=2.5^{\star} e-60\right)$ and so on. Assessment of the immune microenvironment in KIRC

To evaluate the potential relationship between MT1G, LAMP2 and MIOX expression and immune infiltration levels in KIRC, we conducted the following analysis by using TIMER. First, we showed that several immune cell infiltration levels seemed to associate with altered MT1G, LAMP2 and MIOX gene copy numbers, including B cells, CD4+ T cells, CD8+ T cells, macrophages, neutrophil and dendritic cells (DCs) in KIRC (Figure 7D-F). Then, we showed that MT1G, LAMP2 and MIOX expression has markedly positively correlated with infiltrating levels of B cells, CD4+ T cells, CD8+ T cells, macrophages, neutrophil and dendritic cells (DCs) in KIRC (Figure 7A-C). These results suggest that tumor-infiltrating immune cells may have an influence on the clinical outcome of MT1G, LAMP2 and MIOX in KIRC.

The KIRC population in the TCGA-KIRC database was divided into ferroptosis-related-DEGs low expression group (G1) andferroptosis-related-DEGs high expression group (G2), and the correlation between the expression 
of ferroptosis-related-DEGs and immune-infiltrating cells was analyzed. The results show that MT1G, LAMP2 and MIOX were highly correlated with the expression levels of a variety of immune infiltrating cells (Figure 8).

\section{Relationship between methylation and expression of MT1G, LAMP2 and MIOX}

we also explored the correlation of MT1G, LAMP2 and MIOX expression levels and their methylation status. Normalized mRNA expression data with corresponding patients' information were obtained from TCGA-KIRC. The difference of expression of methylation of m6A related genes in KIRC tissues with high and low MT1G, LAMP2 and MIOX was shown in Figure 9A-C. The results suggest thatmethylation of m6A may have an influence on the MT1G, LAMP2 and MIOX in KIRC.

\section{TF-gene interactions and TF-miRNA coregulatory network}

TF-gene interactions were collected using NetworkAnalyst. For MT1G, LAMP2 and MIOX, the TF-genes were identified. TF regulators' interaction with MT1G, LAMP2 and MIOX is visualized in Figure 10A. The network contains 46 nodes and 49 edges. LAMP2 is regulated by 6 TF-genes and MT1G is regulated by 16 TF-genes. MIOX is regulated by 27 TF-genes. These TF-genes regulate more than one common DEGs ofthe network, which indicates high interaction of the TF-genes with common DEGs.

TF-miRNA coregulatory network is generated using NetworkAnalyst. The analysis of the TF-miRNA coregulatory network delivers miRNAs and TFs interaction with the common DEGs. This interaction can be the reason for regulating the expression of the DEGs. The network created for TF-miRNA coregulatory network comprises 62 nodes and 61 edges. Figure 10B dispenses TF-miRNA coregulatory network.

\section{Discussion}

According to the cancer statistics report, more than 65000 patients are diagnosed with RCC, and nearly 15000 deaths happened in RCC patients in USA every year ${ }^{[33]}$. About one third of patients with localized RCC recurred or had metastases after surgical treatment ${ }^{[34]}$. Currently, the prognosis of KIRC is unsatisfactory, with tumor recurrence in about $30 \%$ of patients who are considered disease-free. Since sensitive biomarkers for KIRC have not been identified, we hope to explore and identify effective therapeutic targets and useful prognostic biomarkers through the molecular mechanisms of KIRC occurrence and progression.

Selective induction of cancer cell death is the most effective anticancer therapy ${ }^{[35-36]}$. Increasing evidence has shown that ferroptosis, a recently discovered type of programmed cell death, plays a crucial role in tumorigenesis and cancer therapeutics efficacy. Wang et al ${ }^{[37]}$ elucidated that the cell ferroptosis was regulated by CD8+ T cells and, in turn, can influence the efficacy of cancer immunotherapy. Thus, understanding the relationship between ferroptosis and the complex tumor immune microenvironment in KIRC may help identify novel biomarkers for prognosis and targeted therapy. Currently, surgical resection, chemotherapy, and radiotherapy are the three primary treatments for patients with KIRC. Although surgical resection is considered an effective treatment for improving the survival of early-stage patients, the treatment for many patients with advanced-stage disease is not satisfactory. Cancer immunotherapy provides novel therapeutic options for the comprehensive treatment of advanced resectable KIRC. 
In the present study, we focused on ferroptosis-related genes and investigated their influence on prognosis. We aimed to understand the relationship between the ferroptosis-related prognostic model and the immune microenvironment to further identify potential biomarkers for prognosis detection and target therapy. First, we analyzed DEGs from the GEO: GSE168845, GSE105261, and GSE11151 datasets, assessed intersection with a validated gene set of ferroptosis obtained from the FerrDb database, and found 17 ferroptosis-related genes were received. Of note, we did not take the intersection of four datasets because few genes were obtained, but we summarized the intersection of each GEO dataset and a validated gene set of ferroptosis. Then, functional analysis was performed based on the 17 ferroptosis-related genes, and this revealed that these genes were associated with the immune system process. Univariate Cox and multivariate Cox regression analyses were used to identify the ferroptosis genes with poor prognosis and build a ferroptosis-based prognostic model. Finally, MT1G, LAMP2 and MIOX was considered as prognostic value genes. Subsequently, we studied the immune cell infiltration of the TME using TIMER. We found that the proportions of B cells, CD4+ T cells, CD8+ T cells, macrophages, neutrophil and dendritic cells (DCs) were statistically significant. we also explored the correlation of MT1G, LAMP2 and MIOX expression levels and their methylation status. The results suggest thatmethylation of m6A may have an influence on the MT1G, LAMP2 and MIOX in KIRC. Meanwhile, the analysis of the TF-gene interactions and TF-miRNA coregulatory network delivers miRNAs and TFs interaction with MT1G, LAMP2 and MIOX.

In 2016, sun et al published an article in the authoritative journal "pathology", which found that MT1G promoted the drug resistance of hepatocellular carcinoma to sorafenib by inhibiting iron death. MT1G is a kind of small molecular protein with highly conserved structure, which can induce metal responsive elements and regulate the expression of related genes by binding with metal ions ${ }^{[38]}$. Lysosomal granule membrane protein (LAMP2) is mainly expressed in placenta, lung and liver. In the past, it was considered to be a structural protein. Recent studies suggest that LAMP2 not only performs the function of structural protein, but also participates in autoimmune diseases, autophagy formation, endosome fusion, cholesterol transport, liver fibrosis and other physiological and pathological processes ${ }^{[39]}$. Myo-inositol oxygenase (MIOX), a renal proximal tubular-specific enzyme, was identified several years ago ${ }^{[40]}$. It catabolizes myo-inositol to d-glucuronate, and plays an important role in renal tubular injury ${ }^{[41]}$. The MIOX promoter includes osmotic response elements, carbohydrate response elements, oxidant response elements, and sterol response elements, and thus MIOX transcription is modulated by oxidant stress, high glucose ambience, and the administration of free fatty acids ${ }^{[42]}$.

In addition to this study, we noticed that Liang et al ${ }^{[43]}$ and Liu et al ${ }^{[44]}$ studied the ferroptosis-related genes in hepatocellular carcinoma and glioma, respectively. Liang et al identified ferroptosis-related genes and built a predictive model for OS. Although they also studied the immune functions of the ferroptosis genes, the approaches we used were different. Additionally, Liu et al studied the ferroptosis-related gene signature to predict the OS of patients with glioma and performed experiments to validate the expression and function of ferroptosis-related genes. As multiple studies have focused on ferroptosis in tumorigenesis and progression, we believe our study may help to provide novel insights into cancer immunotherapy.

\section{Conclusion}


In summary, our study identified a ferroptosis-related gene-based prognostic model that is independently associated with OS. Furthermore, the analysis of the ferroptosis-related prognostic model and immune profiles identifies the relevance of the immune microenvironment in affecting KIRC outcomes

\section{Materials And Methods}

\section{Study Population and Data Acquisition}

The RNA expression data from accession numbers GEO: GSE168845, GSE105261 and GSE11151 which contained normal and tumor tissues were downloaded from the GEO database(https://www.ncbi.nIm.nih.gov/geo). All data were quantile normalized via log2-scale transformation to ensure standardization. The gene symbols that were detected in more than one probe were calculated using their mean expression levels. Additionally, the level three RNA sequencing(RNA-seq) data of KIRC were obtained from TCGA database (https://www.cancer.gov/tcga/).

A total of 149 ferroptosis-related genes, including drivers, suppressors, and markers, obtained from the FerrDb database (http://www.zhounan.org/ferrdb/)29 were selected as candidate genes. This study followed the publication guidelines of the GEO and TCGA databases.

\section{Identification of differentially expressed ferroptosis genes and functional analysis}

GEO:GSE168845, GSE105261 and GSE11151 were used to detect the DEGs between tumor and adjacent normal tissues via the R package "limma" in RStudio (version 1.2.5001), with the following cutoff for adjustment: $p$ value $<0.05$ and $|\log 2 \mathrm{FC}| \geq 1$. The $\mathrm{R}$ package "pheatmap" was applied to visualize the degree range of differences in the three datasets. Then, the ferroptosis-related genes were obtained by taking the intersection of candidate genes and DEGs.

Based on these ferroptosis-related DEGs, GO and KEGG analyses were performed by applying the "clusterProfiler" package. A PPI network for the ferroptosis-related DEGs was constructed with Search Tool for the Retrieval of Interacting Genes(STRING), version $11.0 \mathrm{https}$ ://string-db.org/). We performed MCODE to further reveal the densely connected regions. $p<0.05$ was used as a cutoff value.

\section{Development and validation of the pyroptosis-related gene prognostic model}

To assess the prognostic value of the pyroptosis-related genes, we further employed univariate-Cox regression analysis to evaluate the correlations between each gene and survival status in the TCGA cohort. And expression profile of the ferroptosis genes with prognostic value in KIRC samples compared with normal tissues was analyzed.

To prevent omissions, we further employed multivariate-Cox regression analysis to evaluate the correlations between each gene and survival status in the TCGA cohort. We set 0.2 as the cut-off P-value, and 7 survivalrelated genes were identified for further analysis. The LASSO Cox regression model (R package "glmnet") was then utilized to narrow down the candidate genes and to develop the prognostic model. Ultimately, the three genes and their coefficients were retained, and the penalty parameter $(\lambda)$ was decided by the minimum criteria. The risk score was calculated after centralization and standardization (applying the "scale" function in R) of 
the TCGA expression data, and the risk score formula was as follows: Risk Score $=\sum 7 \mathrm{XXixYi} \mathrm{(X:} \mathrm{coefficients,} \mathrm{Y:}$ gene expression level). The TCGA KIRC patients were divided into low- and high-risk subgroups according to the median risk score, and the OS time was compared between the two subgroups via Kaplan-Meier analysis. PCA based on the 3-gene signature was performed by the "prcomp" function in the "stats" R package. The "survival", "survminer" and "timeROC" R packages were employed to perform a 1-, 3- and 5-year ROC curve analysis.

\section{Clinical relevance investigation and a nomogram construction}

To study the correlation between the ferroptosis-related prognostic model and clinicopathologic factors, including $T$ stage, laterality and pathological stage, independent $t$ tests were applied.

Furthermore, a nomogram, which provided the survival probability of a specific outcome, was designed to integrate the risk score of the model as a prognostic factor to evaluate the predictive probability of $1-, 3-$, and 5year $\mathrm{OS}^{[26]}$. A calibration curve depicting the 3-year OS was plotted to visualize the observed rates against the nomogram-predicted probabilities. The R package "rms" was used to plot the nomogram and calibration curves.

\section{Immune infiltrations analysis}

The immune infiltrations analysis of MT1G, LAMP2 and MIOX in RCC was analyzed using TIMER ${ }^{[27]}$. TIMER was also used to evaluate the correlation between genes and the infiltration of immune cells and investigate the association between three ferroptosis genes copy number and immune cell infiltration levels.

\section{Relationship between ferroptosis-related-DEGs and immune microenvironment}

Using xCell algorithm in "immunedeconv" $\mathrm{R}$ package, the relationship between ferroptosis-related-DEGs expression levels and immune cells was analyzed was analyzed in "ggplot2" R package.

\section{Methylation of m6A analysis}

The level three RNA sequencing(RNA-seq) data of KIRC were obtained from TCGA database (https://www.cancer.gov/tcga/). The difference of expression of methylation of m6A related genes(YTHDF1, YTHDF2, HNRNPC, IGF2BP1, IGF2BP2, RBMX, HNRNPA2B1, FTO and ALKBH5) in KIRC tissues with high and low MT1G, LAMP2 and MIOX gene expression was analyzed by R package.

\section{TF-gene interaction and TF-miRNA coregulatory network analysis}

TF-gene gene interaction with MT1G, LAMP2 and MIOX evaluates the outcome of TF on functional pathways and expression levels of the genes ${ }^{[28]}$. NetworkAnalyst (https://www.networkanalyst.ca/) platform is used to identify TF-gene interaction with MT1G, LAMP2 and MIOX. NetworkAnalyst is a comprehensive web platform

for performing geneexpression for numerous species and also enables them toperform meta-analysis ${ }^{[29]}$. The network produced for the TF-gene interaction network is obtained from the ENCODE(https://www.encodeproject.org/) database which is includedin the NetworkAnalyst platform. 
Interactions for TF-miRNA coregulatory were collected from the RegNetwork repository ${ }^{[30]}$ which assists to detect miRNAs and regulatory TFs that regulate MT1G, LAMP2 and MIOX of interest at the post-transcriptional and transcriptional level. TF-miRNA coregulatory network was visualized using NetworkAnalyst.

NetworkAnalyst assists researchers in the easiest way to navigate complex datasets to identify biological features and functions which leads to effective biological hypothesis ${ }^{[31]}$.

\section{Statistical analysis}

Univariate and multivariate Cox regression analyses were conducted via R package "survival"[32], along with hazard ratios (HRs) and 95\% confdence intervals (Cls). Moreover, the diference of various clinical factors was compared by the independent $t$ test. A P<0.05 indicated statistical signifcance.

\section{Abbreviations}

kidney renal clear cell carcinoma (KIRC)

The Cancer Genome Atlas (TCGA)

area under the curve (AUC)

Renal cell carcinoma(RCC)

Lysosomal granule membrane protein (LAMP2)

\section{Declarations}

Ethics approval and consent to participate: Our article is bioinformatics analysis. We do not need ethics approval and consent to participate

Consent for publication: All authors agree for publication.

Availability of data and material: Publicly available datasets were analyzed in this study. This data can be found here: https://tcga.xenahubs.net

Competing interests: There is no conflicts of interest.

Funding: This study was funded by The National Natural Science Foundation of China (No. 81872089, 81370849, 81672551, 81300472, 81070592, 81202268, 81202034), Natural Science Foundation of Jiangsu Province (BK20161434, BL2013032, BK20150642 and BK2012336), Six talent peaks project in Jiangsu Province,

Jiangsu Provincial Medical Innovation Team (CXTDA2017025), The National Key Research and Development Program of China(SQ2017YFSF090096), Jiangsu Provincial Key Research and Development Program(BE2019751), Innovative Team of Jiangsu Provincial (2017ZXKJQWO7), Jiangsu Provincial Medical Talent (ZDRCA2016080). 
Authors' contributions: Ke-Hao Pan and Yi-Fan Liu is the co-first author. Bin Xu and Ming Chen is the cocorresponding author.

Acknowledgements: None

\section{References}

[1]Siegel R L, Miller K D , Jemal A. Cancer statistics, 2020[J]. CA: A Cancer Journal for Clinicians, 2020, 70(1). [2]Motzer R J , Jonasch E , Agarwal N , et al. Kidney cancer, version 3.2015[J]. J Natl Compr Canc Netw, 2015, 13(2):151-159.

[3]Tsivian M, Moreira DM, Caso JR, Mouraviev V, Polascik TJ. Cigarette smoking is associated with advanced renal cell carcinoma. J Clin Oncol. 2011;29(15):2027-2031. doi: 10.1200/JC0.2010.30.9484.

[4]Pischon T, Lahmann PH, Boeing H, Tjonneland A, Halkjaer J, Overvad K, Klipstein-Grobusch K. et al. Body size and risk of renal cell carcinoma in the European Prospective Investigation into Cancer and Nutrition (EPIC) Int J Cancer. 2006;118(3):728-738. doi: 10.1002/ijc.21398.

[5]Wozniak MB, Brennan P, Brenner DR, Overvad K, Olsen A, Tjonneland A, Boutron-Ruault MC. et al. Alcohol consumption and the risk of renal cancers in the European prospective investigation into cancer and nutrition (EPIC) Int J Cancer. 2015;137(8):1953-1966. doi: 10.1002/ijc.29559.

[6]Weikert S, Boeing H, Pischon T, Weikert C, Olsen A, Tjonneland A, Overvad K. et al. Blood pressure and risk of renal cell carcinoma in the European prospective investigation into cancer and nutrition. Am J Epidemiol. 2008;167(4):438-446. doi: 10.1093/aje/kwm321

[7]Moore LE, Boffetta P, Karami S, Brennan P, Stewart PS, Hung R, Zaridze D. et al. Occupational trichloroethylene exposure and renal carcinoma risk: evidence of genetic susceptibility by reductive metabolism gene variants. Cancer Res. 2010;70(16):6527-6536. doi: 10.1158/0008-5472.CAN-09-4167.

[8]Choueiri TK, Je Y, Cho E. Analgesic use and the risk of kidney cancer: a meta-analysis of epidemiologic studies. Int J Cancer. 2014;134(2):384-396. doi: 10.1002/ijc.28093.

[9]Klatte T , Rossi S H , Stewart G D. Prognostic factors and prognostic models for renal cell carcinoma: a literature review[J]. World Journal of Urology, 2018.

[10]Lucca I, de Martino M, Hofbauer SL et al (2015) Comparison of the prognostic value of pretreatment measurements of systemic infammatory response in patients undergoing curative resection of clear cell renal cell carcinoma. World J Urol 33:2045-2052

[11]Haas NB, Manola J, Uzzo RG et al (2016) Adjuvant sunitinib or sorafenib for high-risk, non-metastatic renalcell carcinoma (ECOG-ACRIN E2805): a double-blind, placebo-controlled, randomised, phase 3 trial. Lancet 387:2008-2016 
[12]Sung, H. et al. "Global cancer statistics 2020: GLOBOCAN estimates of incidence and mortality worldwide for 36 cancers in 185 countries." CA Cancer J. Clin. https://doi.org/10.3322/caac.21660 (2021).

[13]FUCHS Y, STELLER H. Programmed cell death in animal development and disease. Cell, 2011,147 (4): $742-$ 758.

[14]STOCKWELLBR, FRIEDMANN ANGELIJP, BAYIR H, et al. Ferroptosis: aregulated cell death Nexuslinking metabolism, redox biology, and disease. Cell, 2017,171(2): 273-285.

[15]ALIM I, CAULFIELD J T, CHEN Y X, et al. Selenium drives a transcriptional adaptive program to block ferroptosis and treat stroke. Cell, 2019,177( 5): 1262-1279.

[16]WENZEL S E, TYURINA Y Y, ZHAO J M, et al. PEBP1 wardens ferroptosis by enabling lipoxygenase generation of lipid death signals. Cell, 2017,171 (3): 628-641.

[17]JIANGL, KONN, LI T, et al. Ferroptosis as a p53- mediated activity during tumour suppression. Nature, 2015,520(7545): 57-62.

[18]ZHANGY, SHIJ, LIU X, et al. BAP1 links metabolic regulation of ferroptosis to tumour suppression. Nat Cell Biol, 2018,20(10): 1181-1192.

[19]HANGAUER M J, VISWANATHAN V S, RYAN M J, et al. Drug-tolerant persister cancer cells are vulnera-ble to GPX4 inhibition. Nature, 2017,551(7679): 247-250.

[20]VISWANATHAN V S, RYAN M J, DHRUV H D, et al. Dependency of a therapy-resistant state of cancer cells on a lipid peroxidase pathway. Nature, 2017, 547(7664): 453-457.

[21]WANG W M, GREEN M, CHOI J E, et al. CD8+ T cells regulate tumour ferroptosis during cancer immunotherapy. Nature, 2019,569(7755): 270-274.

[22]CONRAD M, LORENZ, M, PRONETH B. Targeting ferroptosis: new hope for as-yet-incurable diseases. Trends Mol Med,2020,27(2):113-122.

[23]ELGENDY S M, ALYAMMAHI S K, ALHAMAD D W, et al. Ferroptosis: an emerging approach for targeting cancer stem cells and drug resistance. Crit Rev Oncol Hematol,2020,155:103095.

[24]ALA M, MOHAMMAD JAFARI R, AGBELE A T, et al. Sumatriptan alleviates radiation-induced oral mucositis in rats by inhibition of NF-kB and ERK activation, prevention of TNF- $a$ and ROS release. Arch Oral Biol,2020,119:104919.

[25]Friedmann Angeli, J. P., Krysko, D. V., and Conrad, M. (2019). Ferroptosis at the crossroads of canceracquired drug resistance and immune evasion. Nat. Rev. Cancer 19, 405-414. doi: 10.1038/s41568-019-0149-1.

[26]Ferrone C.R., Kattan M.W., Tomlinson J.S., Thayer S.P., Brennan M.F., Warshaw A.L. Validation of a postresection pancreatic adenocarcinoma nomogram for disease-specific survival. J Clin Oncol. 2005;23:75297535 . 
[27]Li T, Fan J, Wang B, Traugh N, Chen Q, Liu JS, et al. TIMER: A web server for comprehensive analysis of tumor-infiltrating immune cells. Cancer Res. (2017)77: e108-10. doi: 10.1158/0008-5472.

[28]Ye Z, Wang F, Yan F, et al. Bioinformatic identification of candidate biomarkers and related transcription factors in nasopharyngeal carcinoma. World J Surg Oncol 2019;17(1):60.

[29]Zhou G, Soufan O, Ewald J, et al. NetworkAnalyst 3.0: a visual analytics platform for comprehensive gene expression profiling and meta-analysis. Nucleic Acids Res 2019;47(W1):W234-41.

[30]Liu ZP, Wu C, Miao H, et al. RegNetwork: an integrated database of transcriptional and post-transcriptional regulatory networks in human and mouse. Database. 2015;2015:bav095.

[31]Xia J, Gill EE, Hancock RE. NetworkAnalyst for statistical,visual and network-based meta-analysis of gene expression data. Nat Protoc 2015;10(6):823.

[32]Therneau TM (2015) A package for survival analysis in S. Version 2.38. CRAN website-https://cran.rproject.org/package=survival.

[33]Siegel RL, Miller KD, Jemal A. Cancer statistics, 2018. CA Cancer J Clin. 2018, 68(1): 7-30.

[34]Brierley JD, Gospodarowicz MK, Wittekind C, et al. TNM Classification of Malignant Tumours. [M]. 8th ed. Chichester: Wiley-Blackwell. 2016: 187-210.

[35]Phan T, Nguyen V.H, A lincourt Salazar M, Wong P, Diamond D.J, Yim J.H, Melstrom L.G. Inhibition of Autophagy Amplifies Baicalein-Induced Apoptosis in Human Colorectal Cancer. Mol Ther Oncolytics. 2020;19:17.

[36]Yang B.C, Leung P.S. Irisin Is a Positive Regulator for Ferroptosis in Pancreatic Cancer. Mol Ther Oncolytics. 2020;18:457-466.

[37]Wang W, Green M, Choi J.E, et al. CD8+ T cells regulate tumour ferroptosis during cancer immunotherapy. Nature. 2019;569:270-274.

[38]SUN X F, NIU X H, CHEN R C, et al. Metallothionein-1G facilitates sorafenib resistance through inhibition of ferroptosis. Hepatology,2016,64(2):488-500.

[39]YuanfengLiu, Dongting Zhao, Wenyi Peng, et al. Atmospheric PM2.5 blocking up autophagic flux in HUVECs via inhibiting Sntaxin-17 and LAMP2. Ecotoxicology and Environmental Safety. Volume 208, 2021.

[40]Nayak B, et al. Modulation of renal-specific oxidoreductase/myo-inositol oxygenase by high-glucose ambience. Proc Natl Acad Sci USA. 2005;102(50):17952-17957.

[41]Sharma I, Dutta RK, Singh NK, Kanwar YS. High glucose-induced hypomethylation promotes binding of Sp1 to Myo-inositol oxygenase: implication in the pathobiology of diabetic tubulopathy. Am J Pathol. 2017;187(4):724-739. 
[42]Sharma I, Tupe RS, Wallner AK, Kanwar YS. Contribution of myo-inositol oxygenase in AGE: RAGE-mediated renal tubulointerstitial injury in the context of diabetic nephropathy. Am J Physiol Renal Physiol.

2018;314(1):F107-F121.

[43]Liang J.Y, Wang D.S, Lin H.C, Chen X.X, Yang H, Zheng Y, Li Y.H. A Novel Ferroptosis-related Gene Signature for Overall Survival Prediction in Patients with Hepatocellular Carcinoma. Int J Biol Sci. 2020;16:2430-2441

[44]Liu H.J, Hu H.M, Li G.Z, Zhang Y, Wu F, Liu X, Wang K.Y, Zhang C.B, Jiang T. Ferroptosis-Related Gene Signature Predicts Glioma Cell Death and Glioma Patient Progression. Front. Cell Dev. Biol. 2020;8:538.

\section{Tables}

Table 1. The information of datasets from the GEO database

\begin{tabular}{lllll} 
Accession number & Platform & Samples & Experiment type & Organism \\
\hline GEO: GSE168845 & GPL21185 & 8 & expression profiling by array & Homo sapiens \\
\hline GEO: GSE105261 & GPL10558 & 44 & expression profiling by array & Homo sapiens \\
\hline GEO: GSE11151 & GPL570 & 58 & expression profiling by array & Homo sapiens
\end{tabular}

Table 2. GO enrichments. Bioinformatics analysis of the dysregulated ferroptosis genes in KIRC. 


\begin{tabular}{|c|c|c|c|c|c|c|c|}
\hline ONTOLOGY & ID & Description & GeneRatio & BgRatio & pvalue & p.adjust & qvalue \\
\hline $\mathrm{BP}$ & GO:0048548 & $\begin{array}{l}\text { regulation of } \\
\text { pinocytosis }\end{array}$ & $2 / 16$ & $11 / 18670$ & $\begin{array}{l}3.77 \mathrm{e}- \\
05\end{array}$ & 0.018 & 0.011 \\
\hline BP & GO:0072584 & $\begin{array}{l}\text { caveolin- } \\
\text { mediated } \\
\text { endocytosis }\end{array}$ & $2 / 16$ & $11 / 18670$ & $\begin{array}{l}3.77 e- \\
05\end{array}$ & 0.018 & 0.011 \\
\hline $\mathrm{BP}$ & GO:0051775 & $\begin{array}{l}\text { response to } \\
\text { redox state }\end{array}$ & $2 / 16$ & $14 / 18670$ & $\begin{array}{l}6.23 e- \\
05\end{array}$ & 0.018 & 0.011 \\
\hline $\mathrm{BP}$ & GO:0043618 & $\begin{array}{l}\text { regulation of } \\
\text { transcription } \\
\text { from RNA } \\
\text { polymerase II } \\
\text { promoter in } \\
\text { response to } \\
\text { stress }\end{array}$ & $3 / 16$ & $121 / 18670$ & $\begin{array}{l}1.40 \mathrm{e}- \\
04\end{array}$ & 0.018 & 0.011 \\
\hline $\mathrm{BP}$ & GO:0006907 & pinocytosis & $2 / 16$ & $21 / 18670$ & $\begin{array}{l}1.43 \mathrm{e}- \\
04\end{array}$ & 0.018 & 0.011 \\
\hline $\mathrm{CC}$ & GO:0031528 & $\begin{array}{l}\text { microvillus } \\
\text { membrane }\end{array}$ & $2 / 16$ & $23 / 19717$ & $\begin{array}{l}1.55 \mathrm{e}- \\
04\end{array}$ & 0.014 & 0.010 \\
\hline $\mathrm{CC}$ & GO:0005811 & lipid droplet & $2 / 16$ & $81 / 19717$ & 0.002 & 0.033 & 0.023 \\
\hline $\mathrm{CC}$ & Go:0045121 & membrane raft & $3 / 16$ & $315 / 19717$ & 0.002 & 0.033 & 0.023 \\
\hline $\mathrm{CC}$ & G0:0098857 & $\begin{array}{l}\text { membrane } \\
\text { microdomain }\end{array}$ & $3 / 16$ & $316 / 19717$ & 0.002 & 0.033 & 0.023 \\
\hline $\mathrm{CC}$ & GO:0005902 & microvillus & $2 / 16$ & 83/19717 & 0.002 & 0.033 & 0.023 \\
\hline MF & GO:0016701 & $\begin{array}{l}\text { oxidoreductase } \\
\text { activity, acting } \\
\text { on single } \\
\text { donors with } \\
\text { incorporation of } \\
\text { molecular } \\
\text { oxygen }\end{array}$ & $2 / 16$ & $28 / 17697$ & $\begin{array}{l}2.86 \mathrm{e} \\
04\end{array}$ & 0.010 & 0.005 \\
\hline MF & GO:0005506 & iron ion binding & $3 / 16$ & $152 / 17697$ & $\begin{array}{l}3.20 \mathrm{e}- \\
04\end{array}$ & 0.010 & 0.005 \\
\hline MF & GO:0030291 & $\begin{array}{l}\text { protein } \\
\text { serine/threonine } \\
\text { kinase inhibitor } \\
\text { activity }\end{array}$ & $2 / 16$ & $32 / 17697$ & $\begin{array}{l}3.74 \mathrm{e}- \\
04\end{array}$ & 0.010 & 0.005 \\
\hline MF & GO:0015485 & $\begin{array}{l}\text { cholesterol } \\
\text { binding }\end{array}$ & $2 / 16$ & 49/17697 & $\begin{array}{l}8.79 \mathrm{e}- \\
04\end{array}$ & 0.018 & 0.009 \\
\hline MF & GO:0032934 & sterol binding & $2 / 16$ & $56 / 17697$ & 0.001 & 0.019 & 0.010 \\
\hline
\end{tabular}

Table 3. Univariate and multivariate Cox regression analyses of the seven ferroptosis genes for OS in the entire TCGA cohort. 


\begin{tabular}{|c|c|c|c|c|c|}
\hline \multirow[t]{2}{*}{ Characteristics } & \multirow[t]{2}{*}{ Total(N) } & \multicolumn{2}{|l|}{ Univariate analysis } & \multicolumn{2}{|l|}{ Multivariate analysis } \\
\hline & & $\begin{array}{l}\text { Hazard ratio }(95 \% \\
\mathrm{Cl})\end{array}$ & $\begin{array}{l}P \\
\text { value }\end{array}$ & $\begin{array}{l}\text { Hazard ratio }(95 \% \\
\mathrm{Cl})\end{array}$ & $\begin{array}{l}\mathrm{P} \\
\text { value }\end{array}$ \\
\hline CDKN2A (Low vs. High) & 539 & $0.734(0.544-0.992)$ & 0.044 & $0.765(0.563-1.038)$ & 0.085 \\
\hline $\begin{array}{l}\text { LINC00472 (Low vs. } \\
\text { High) }\end{array}$ & 539 & $1.557(1.146-2.117)$ & 0.005 & $1.122(0.800-1.574)$ & 0.505 \\
\hline MT1G (Low vs. High) & 539 & $0.592(0.437-0.802)$ & $<0.001$ & $0.666(0.486-0.914)$ & 0.012 \\
\hline PROM2 (Low vs. High) & 539 & $0.558(0.411-0.758)$ & $<0.001$ & $0.641(0.467-0.879)$ & 0.006 \\
\hline CA9 (Low vs. High) & 539 & $1.422(1.051-1.924)$ & 0.022 & $1.356(0.999-1.843)$ & 0.051 \\
\hline LAMP2 (Low vs. High) & 539 & $1.722(1.267-2.339)$ & $<0.001$ & $1.549(1.128-2.128)$ & 0.007 \\
\hline MIOX (Low vs. High) & 539 & 1.959 (1.439-2.667) & $<0.001$ & $1.603(1.158-2.219)$ & 0.004 \\
\hline
\end{tabular}

\section{Figures}




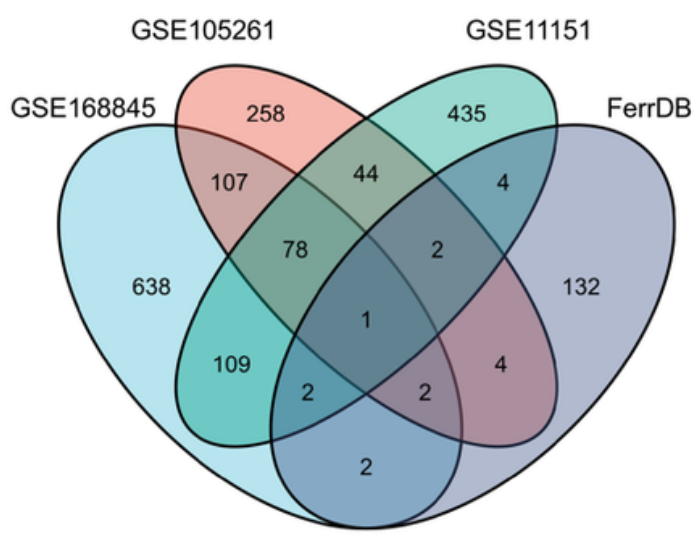

C

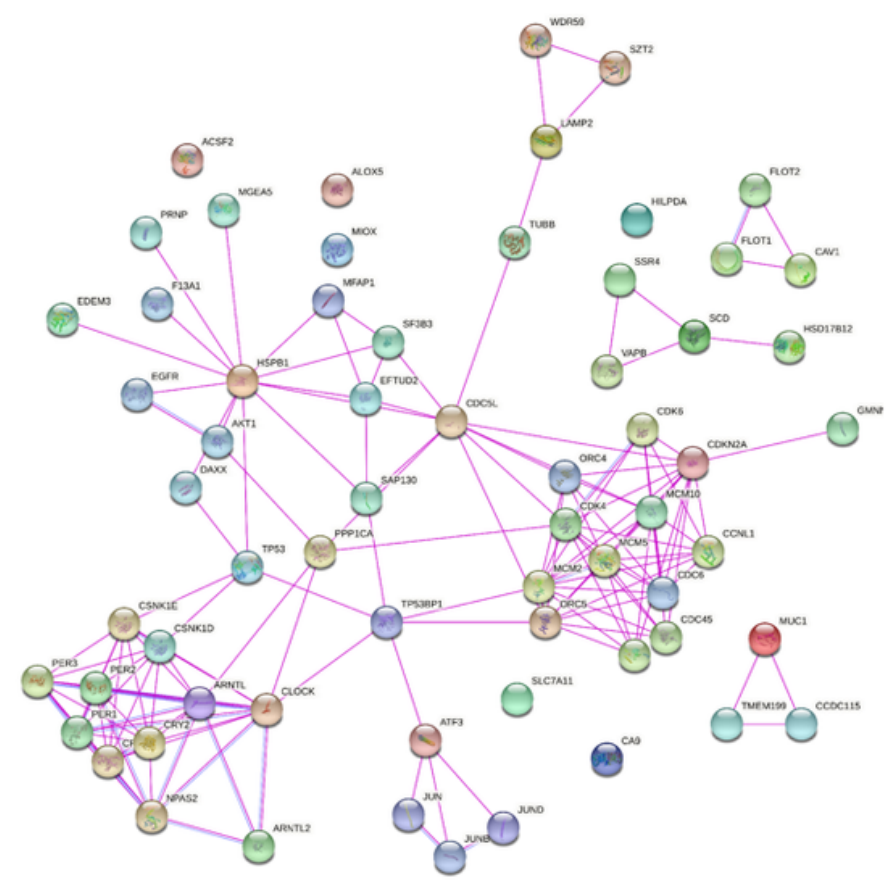

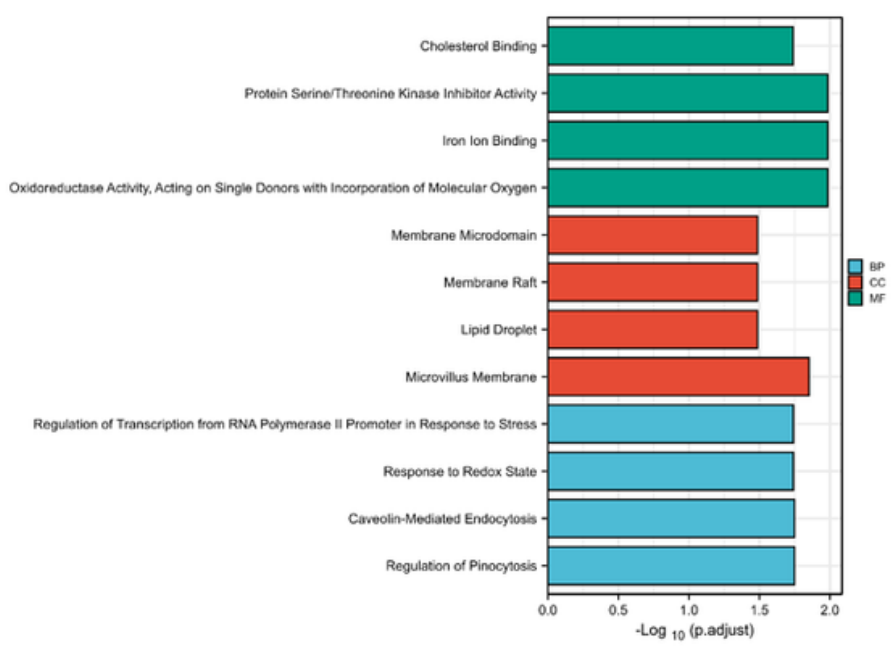

D

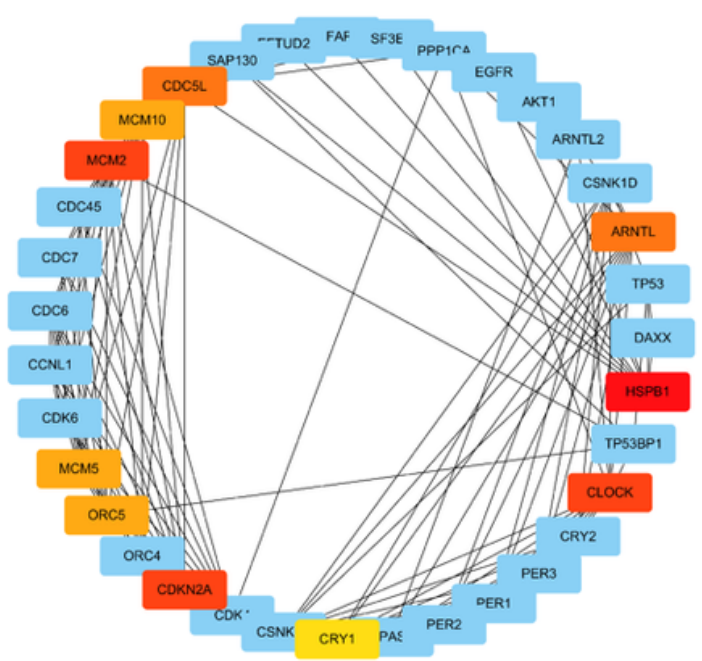

\section{Figure 1}

Overview of the differentially expressed ferroptosis signatures in KIRC

(A) Venn diagram showing the dysregulated ferroptosis genes common to the four datasets. (B) Graph showing the GO analysis. ( $C$ and D) PPI network and MCODE showing the hub genes in the ferroptosis gene set. 


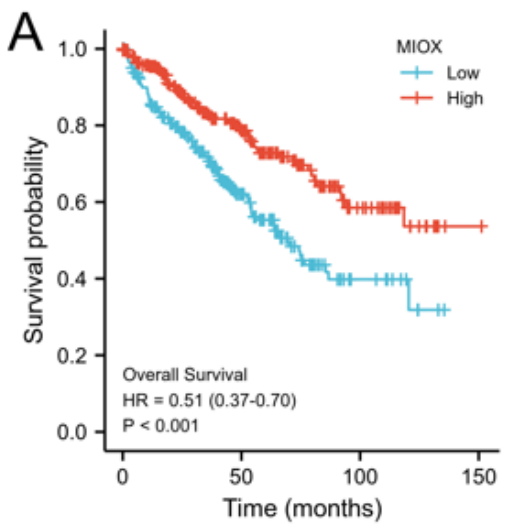

n

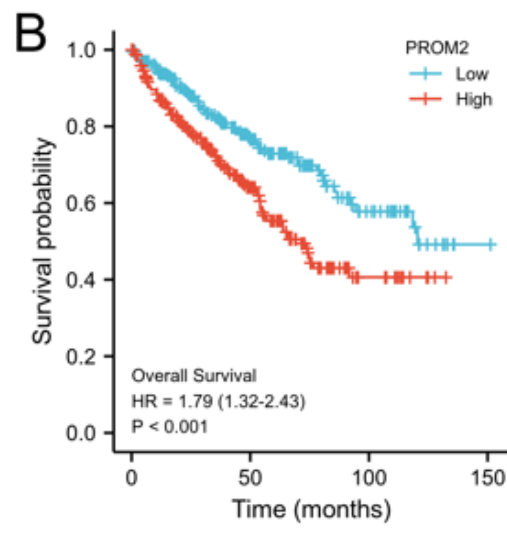

ᄃ

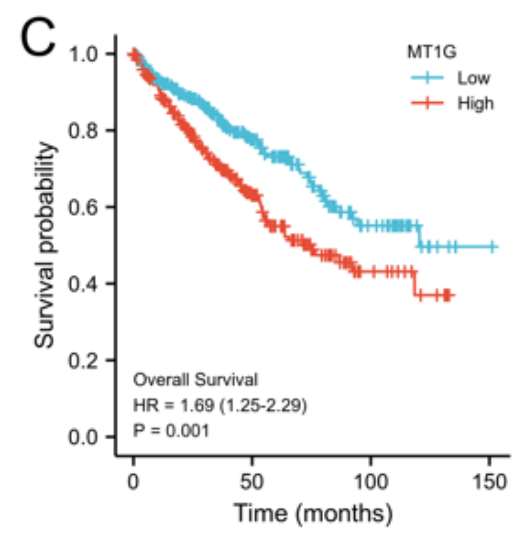

ᄃ

\section{Figure 2}

Kaplan-Meier plots and forests plot of the prognostic ferroptosis signature

G) Kaplan-Meier plots showing the ferroptosis genes with prognostic value. $(\mathrm{H})$ The forest plot showing the results of the univariate Cox regression analyses. 

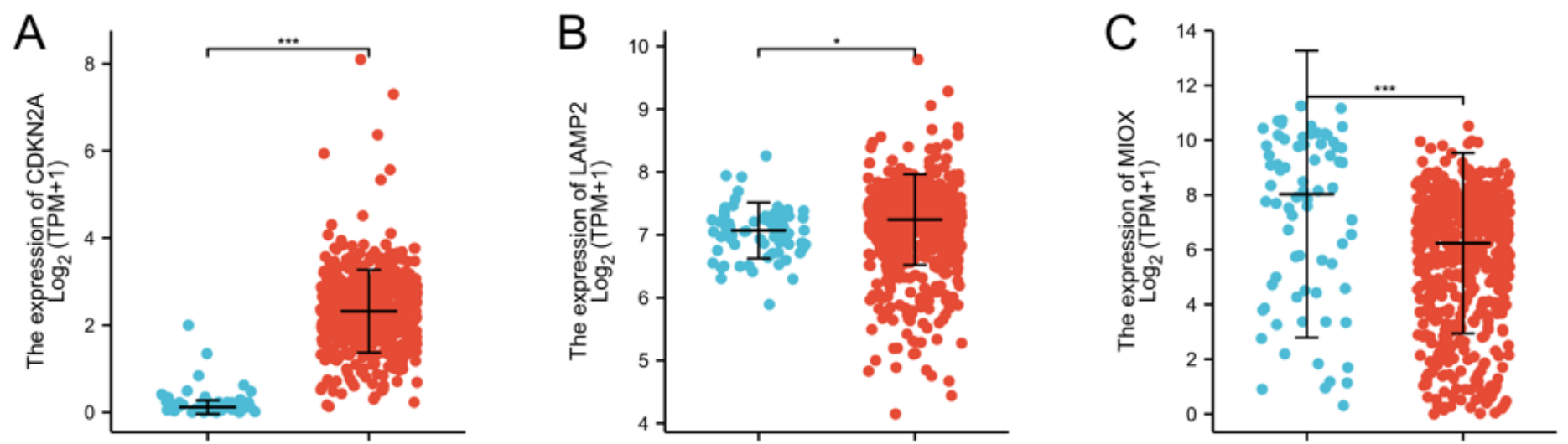

\section{Figure 3}

Expression profile of the ferroptosis genes with prognostic value in KIRC samples compared with normal tissues(A-G)

\section{Figure 4}

The ferroptosis genes accurately predict survival of KIRC patients 
Risk score distribution, survival status, and expression of 3 ferroptosis genes for patients in low-and high-risk groups (A), KM survival analyses (B), time dependent ROC curve analyses (C) in TCGA set.
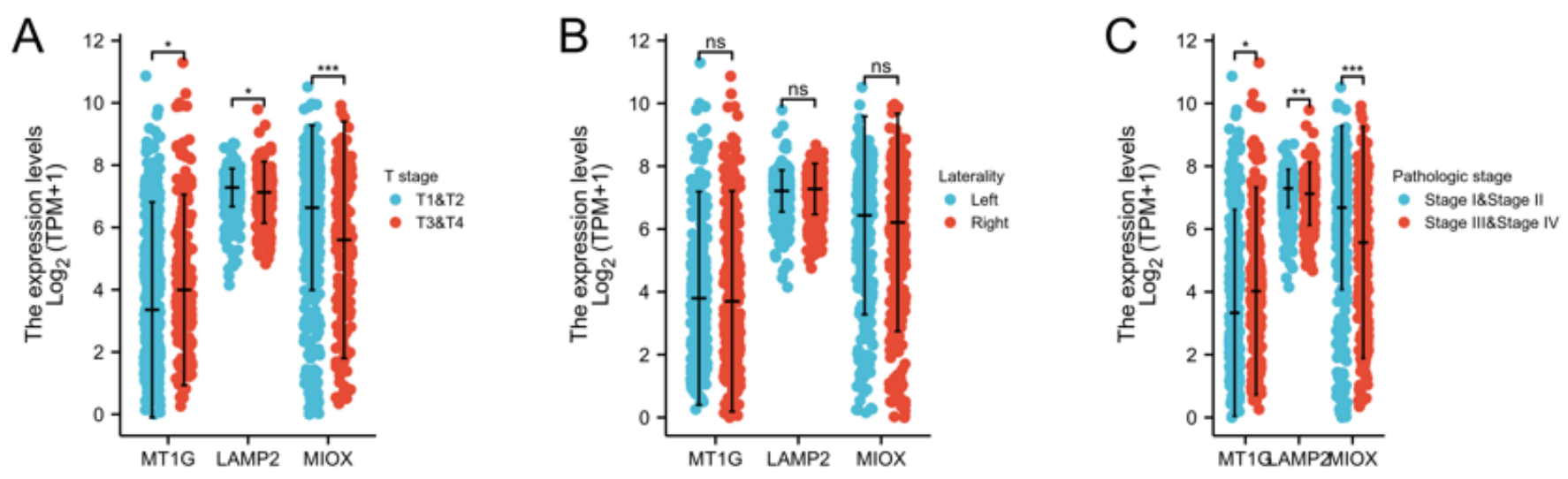

D
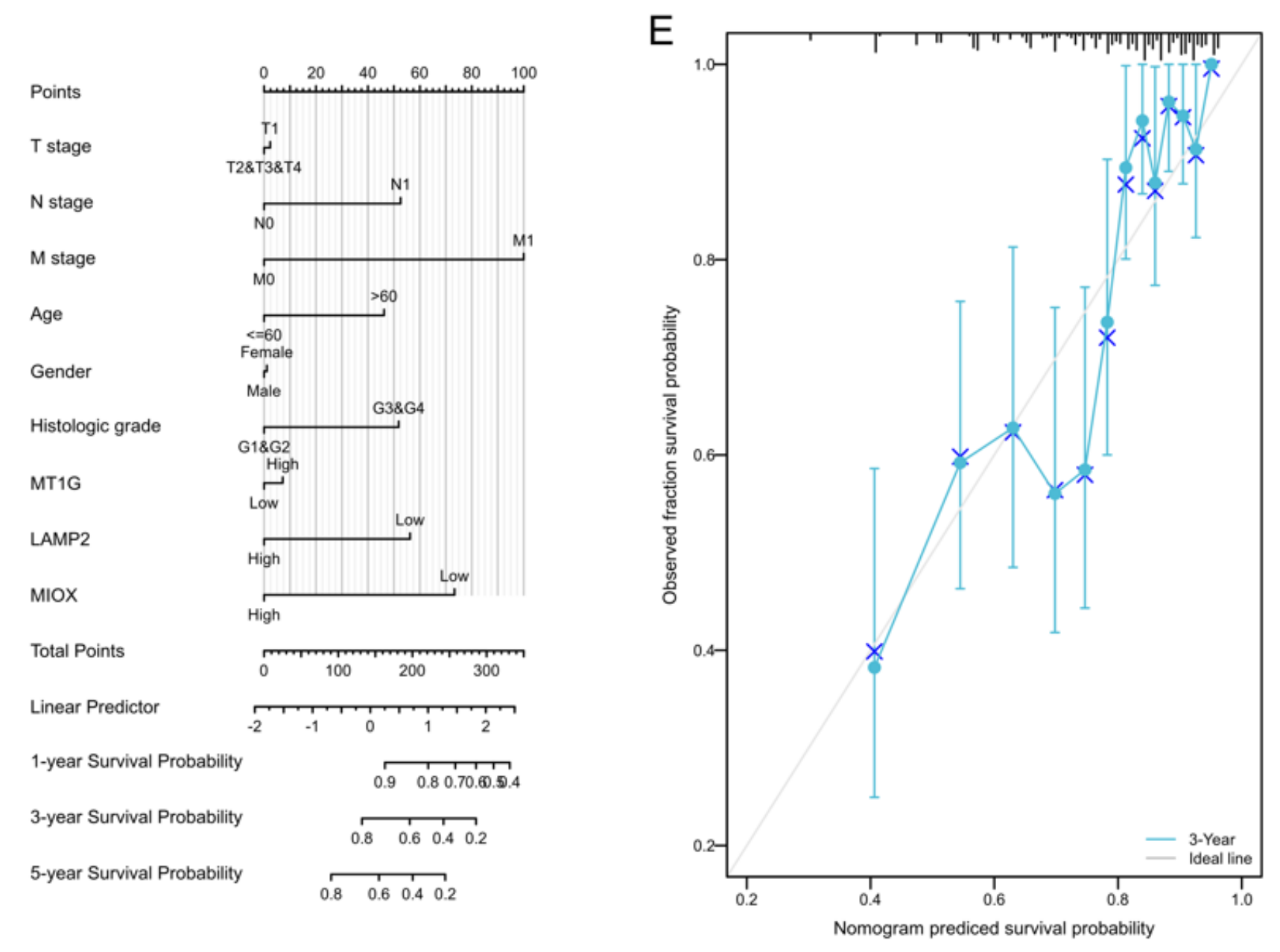

\section{Figure 5}

Three ferroptosis genes significantly correlates with multiple clinicopathological factors in KIRC patients

The relationships between three ferroptosis genes and clinicopathological factors in entire TCGA cohort (A-C). (D) Nomogram for predicting 1-, 3-, and 5-year OS in entire TCGA cohort. (E) Calibration curves of nomogram 
on consistency between predicted and observed 3-year survival in entire TCGA cohort. Dashed line at $45^{\circ}$ implicated a perfect prediction, and the actual performances of our nomogram were shown in blue lines.

Figure 6

Spearman correlation analysis between risk score and immune score.

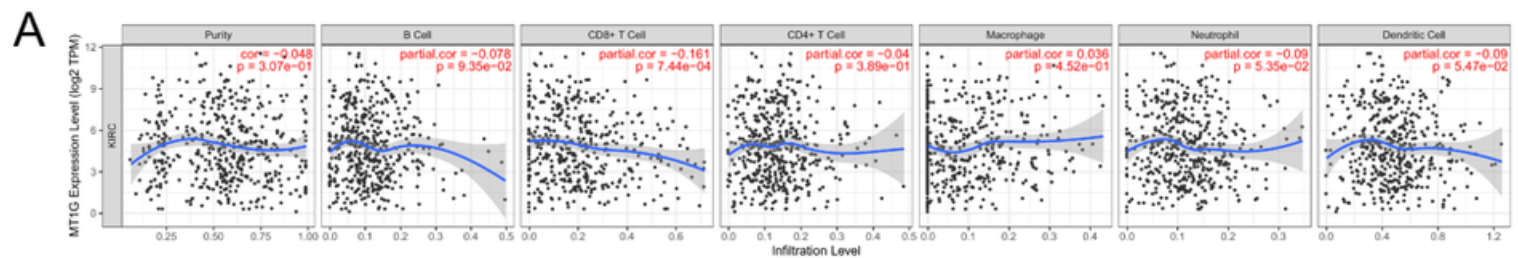

B

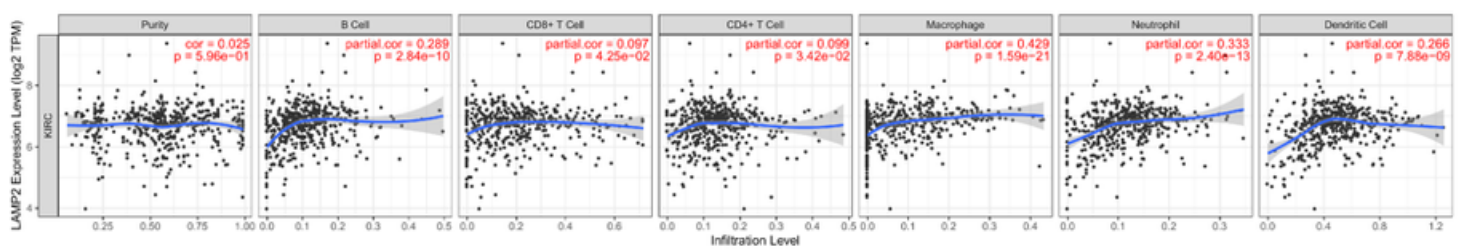

C

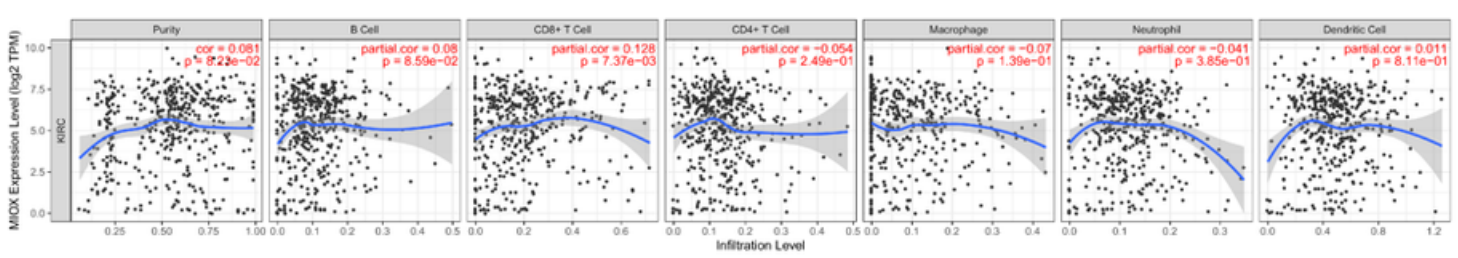

D

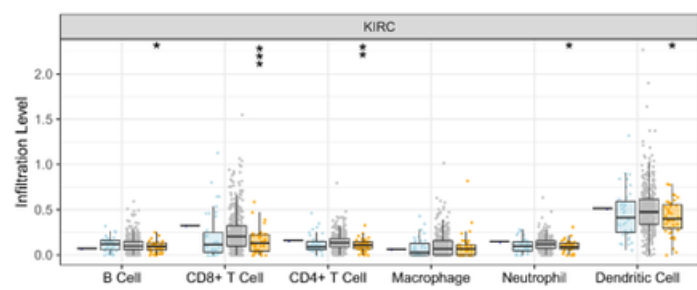

Copy Number

帛 Doep Deleton

帛 DiplididNormal

E

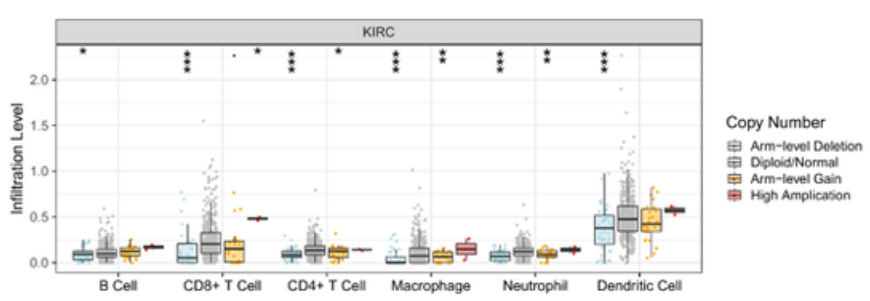

$\mathrm{F}$

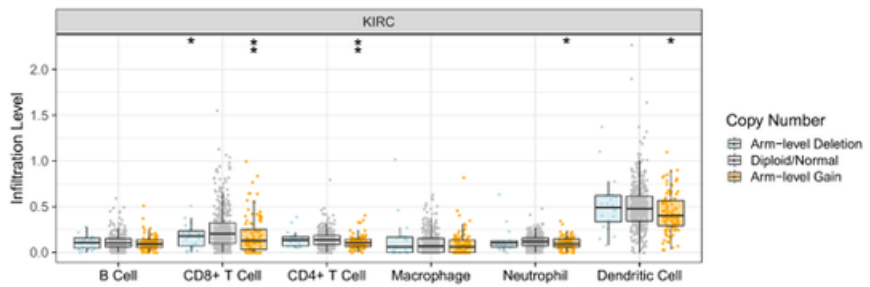

Figure 7 
Three ferroptosis genes expression-MT1G (A), LAMP2 (B) and MIOX (C) is correlated with immune infiltration levels in KIRC. Based on the TIMER database, we detected the correlation of three ferroptosis genes with levels of Cancer associated B cell, Myeloid dendritic cell, CD4+ T cell, Neutrophil, CD8+ T cell, Macrophage. Association between three ferroptosis genes-MT1G (D), LAMP2 (E) and MIOX (F) copy number and immune cell infiltration levels in KIRC cohorts.

A

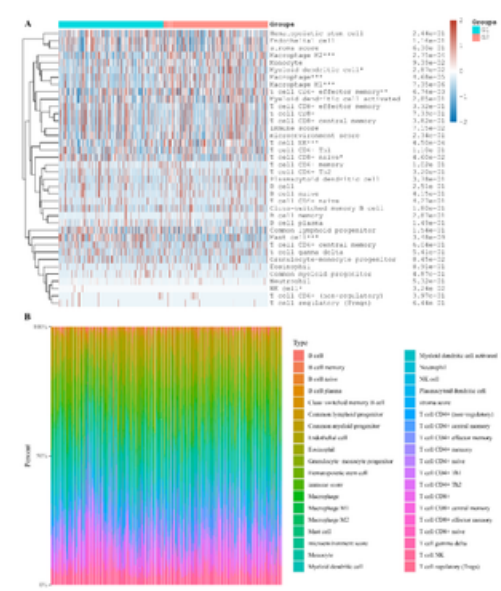

B
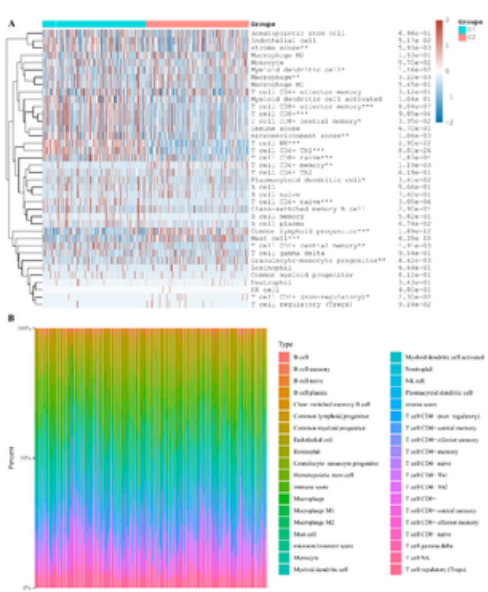

C

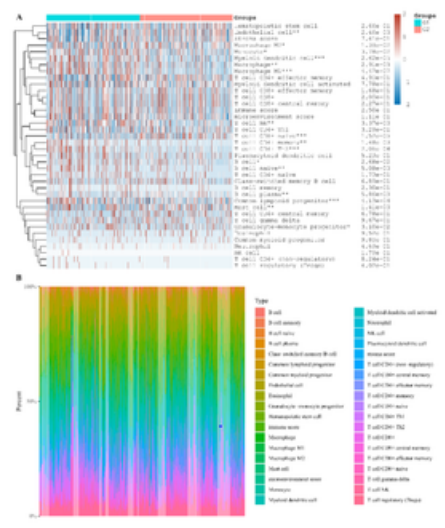

Figure 8 
The difference of expression of immune infiltration cells in KIRC tissues with low and high MT1G (A), LAMP2 (B) and MIOX (C) gene expression. G1 is a low expression group and G2 is a high expression group. ${ }^{*}<0.05$, $\star * p<0.01, * * * p<0.001$.

A
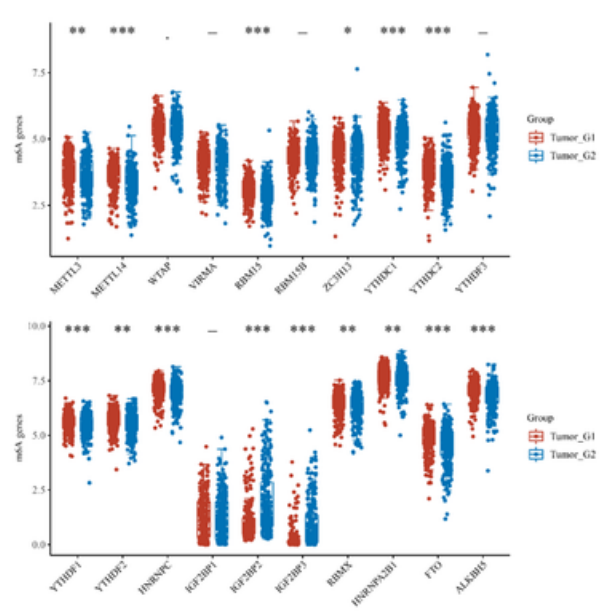

B
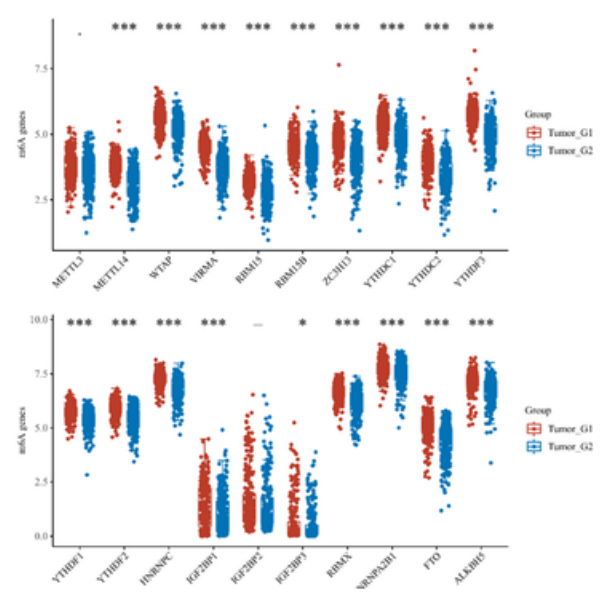

C
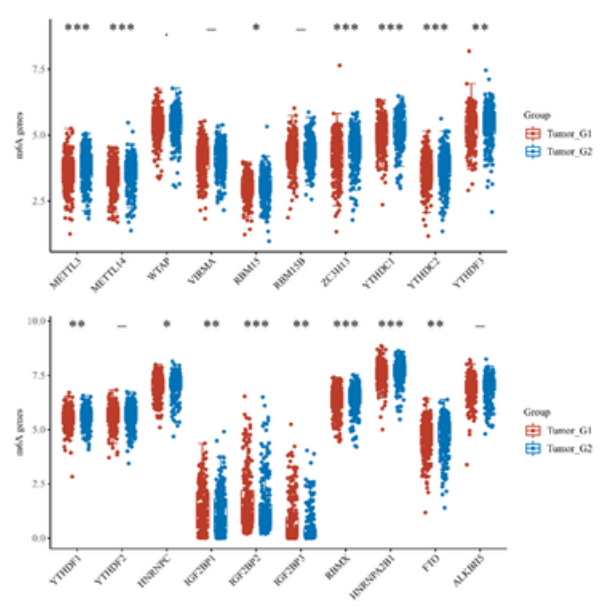

Figure 9

The difference of expression of methylation of m6A related genes in KIRC tissues with high and low MT1G (A), LAMP2 (B)and MIOX (C) gene expression. G1 is a high expression group and G2 is a low expression group. 


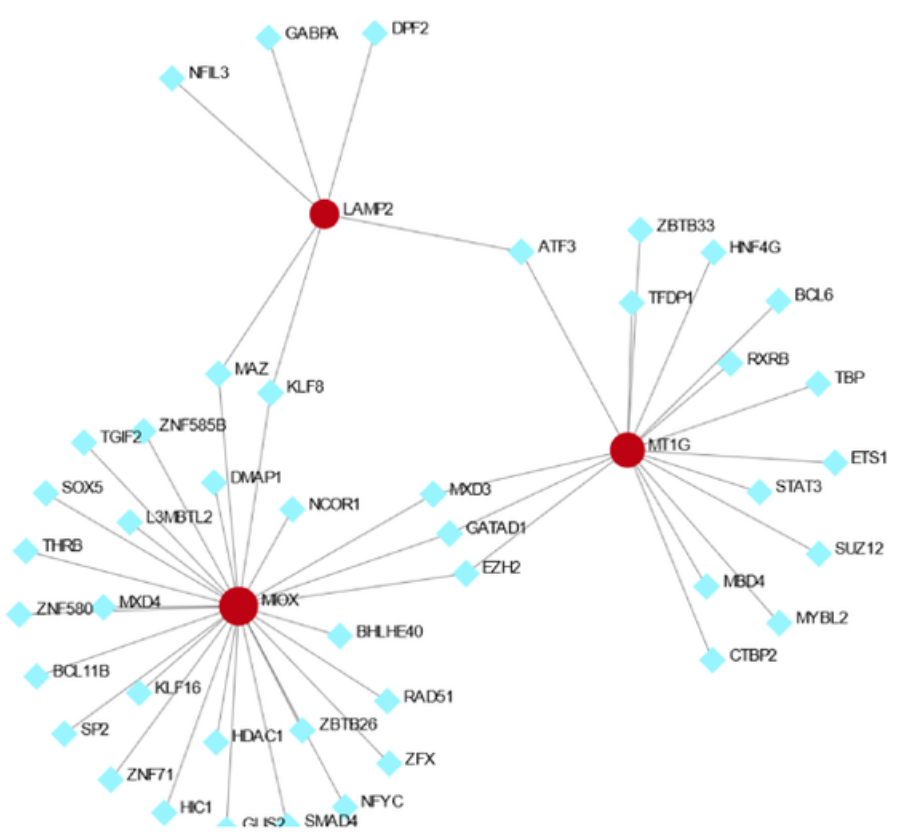

B

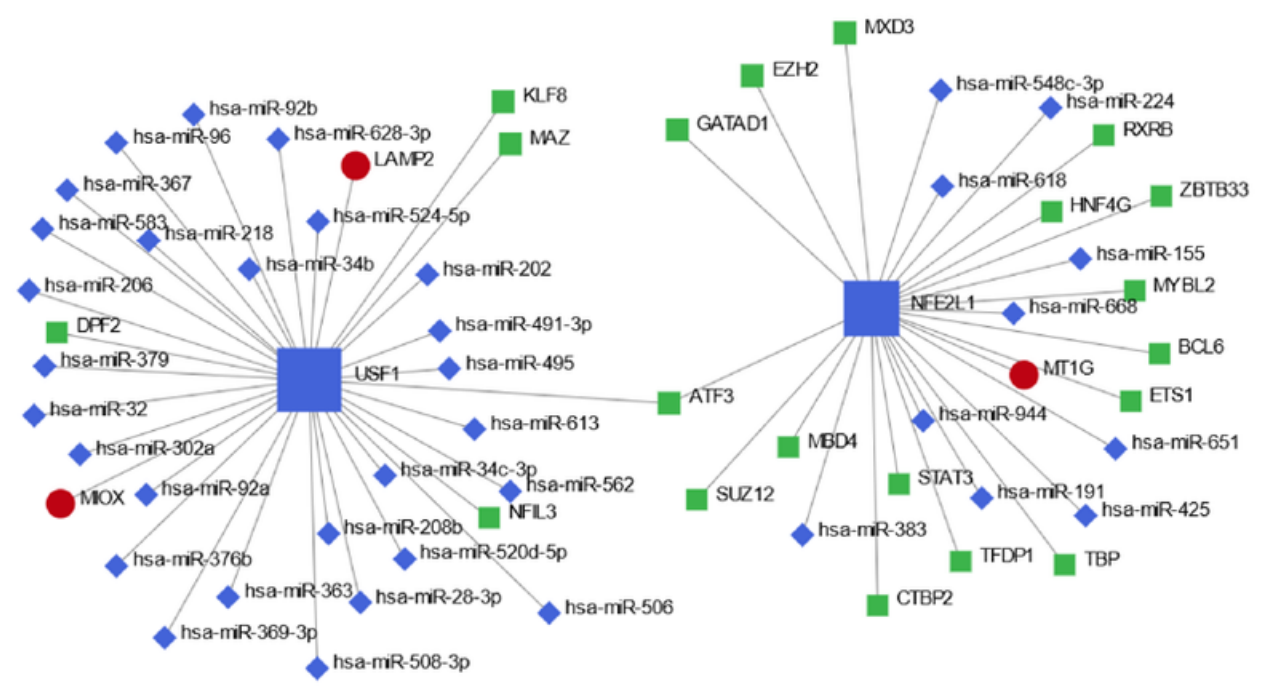

\section{Figure 10}

Network for TF-gene interaction (A) and TF-miRNA(B) coregulatory network with three ferroptosis genes.

(A) The highlighted red color node represents the ferroptosis genes and other nodes represent TF-genes. The network consists of 46 nodes and 49 edges. (B)The network presents the TF-miRNA coregulatory network. The network consists of 62 nodes and 61 edges including 24 TF-genes, 38 miRNA and three differentially expressed genes. The nodes in red color are the differentially expressed genes, a blue node represents miRNA and green nodes indicate TF-genes. 